\title{
Development of the interatrial wall during the ontogenesis of foetuses and children up to one year of age
}

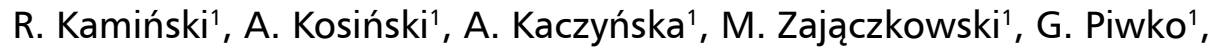 \\ M. Gleinert-Rożek' ${ }^{1}$, T. Gos'², K. Karnecki² \\ 'Department of Clinical Anatomy, Medical University of Gdansk, Poland \\ ${ }^{2}$ Department of Forensic Medicine, Medical University of Gdansk, Poland
}

[Received: 29 October 2019; Accepted: 30 December 2019]

\begin{abstract}
Background: The foramen ovale, present in foetal interatrial septum, plays an important role during foetal life. During delivery, foramen ovale closes and becomes fossa ovalis, starting the pulmonary circulation. The aim of our study was to describe the growth of the interatrial wall and changes in location of the foramen ovale, and fossa ovalis during the ontogenesis in the human hearts.

Materials and methods: The study was performed on post-mortem material obtained from 92 human hearts from $22^{\text {nd }}$ week of foetal life up to 1 year of age, fixed in a $4 \%$ formalin solution.

Results: The interatrial wall size in the studied development period was greater in the horizontal than in the vertical dimension. During ontogenesis up to 1 year old, the anterior and inferior parts of the interatrial wall increased their shares considerably by $8 \%$ and $6 \%$, respectively. The percentage participation of foramen ovale in the interatrial wall construction in the foetal period formed more than $50 \%$ of its size and fairly decreased reaching in infants about 39\%.

Conclusions: Our study demonstrated that during ontogenesis, from the foetal period to infancy, the parts of the interatrial wall increase their dimensions unevenly. The foramen ovale growth is smaller, compared to the rest of the interatrial wall development. On the basis of our data we can assume that the foramen ovale centre tends to be found in the postero-inferior quadrant of the interatrial wall (foetuses) and in postero-superior quadrant of the interatrial wall - in infants. (Folia Morphol 2020; 79, 4: 736-741)
\end{abstract}

Key words: interatrial wall, foramen ovale, fossa ovalis, right atrium

\section{INTRODUCTION}

The foramen ovale (FO), present in foetal atrial septum, plays an important role during foetal life as it allows blood to flow from the inferior vena cava (IVC) directly to left atrium bypassing pulmonary circulation. During delivery, foramen ovale closes and becomes fossa ovalis (fo), starting the pulmonary cir- culation. The process of atrial septation in the human hearts is very sophisticated. At the end of embryonic stage, the primary septum forms the bottom, or flap valve of the fo, with the secondary foramen, recognisable as the FO. The true atrial septum is only the fo and its antero-inferior rim [1]. In patients with congenital heart malformations, most often with

Address for correspondence: A. Kosiński, MD, PhD, Department of Clinical Anatomy, Medical University of Gdansk, ul. Dębinki 1, 80-211 Gdańsk, Poland, tel: +48 58349 1420, e-mail: adam.kosinski@gumed.edu.pl 
the transposition of great arteries, maintaining the functional blood passage within the atrial septum is a matter of life or death of the child. In this case, the closure of foramen ovale may lead to disabled gas exchange and the development of respiratory failure. Then, atrial septostomy is a suggested life-saving procedure in the majority of neonates with the transposition of great arteries $[7,9,10]$.

The aim of our study was to describe the growth of the interatrial wall and changes in location of the $\mathrm{FO}$ and fo during the ontogenesis, in normal, human hearts.

\section{MATERIALS AND METHODS}

Our research project was approved by Independent Research Bioethics Committee of Medical University of Gdansk (IRBC) and achieved permit number: NKBBN/165/2018. Investigation has been conducted according to the principles expressed in the Declaration of Helsinki. The decision of IRBC repeals the need for the consent of donors and their next of kin for the implementation of the research presented in the article. IRBC specifically waived the need for written informed consent from the donors/next of kin. The study was performed on post-mortem material obtained from 92 human hearts from 22 week of foetal life up to 1 year of age, fixed in a $4 \%$ formalin solution. Only those hearts were used for research, which macroscopically showed no developmental anomalies and had no medical history of any chromosomal anomalies or any diseases. Moreover, the hearts included to our study were taken from mothers who had no medical records of chronic diseases like hypertension, diabetes, or renal failure and the pregnancy period was not disturbed, like for example by HELLP syndrome. The available material was divided into three age groups: the first group (1) consisted of 41 hearts between $22^{\text {nd }}$ and $37^{\text {th }}$ week of foetal life $(\mathrm{Hbd})$, (Fig. $\left.1 \mathrm{~A}\right)$ the second group (2) consisted of 30 hearts of neonates - from birth until the end of the $4^{\text {th }}$ week of life (Fig. 1B); the third group (3) accounted for 21 hearts of infants from 1 to 12 months old (Fig. 1C). Due to the very similar dimensions of hearts in the early stages of individual development (until the first year of life), and quite a significant measurement error $(0.5 \mathrm{~mm})$, the test was carried out without taking into account gender. Classical anatomical studies were applied. We opened the right atrium by an incision extending from the orifice of the superior vena cava (SVC) to the orifice of the IVC. We made some meas- urements within the interatrial wall (IAW) viewed from the right atrium according to the schematic diagram showed in Figure 1D. Anterior limit of the IAW was the line running along the right atrio-ventricular ring. Posterior limit of the IAW was the line connecting the dorsal parts of the orifices of the superior and inferior caval veins. Inferior limit of the IAW was the line running from the lower part of the septal cusp of the tricuspid valve (SCTV) to the dorsal part of the IVC orifice. Superior limit of the IAW was the line between the upper part of the SCTV and dorsal part of SVC orifice. Dimension A was the distance from the posterior limit of the IAW to posterior limbus of $\mathrm{FO} / \mathrm{fo}$; dimension B - distance between the anterior and posterior limbus of FO/fo; dimension $\mathrm{C}$ - distance from the anterior limbus of $\mathrm{FO} / \mathrm{fo}$ to the anterior limit of the $I A W$; dimension $D$ - distance from the inferior limit of the IAW to the inferior limbus of $\mathrm{FO} / \mathrm{fo}$; dimension $\mathrm{E}-$ distance between upper and bottom limbus of FO/fo; dimension $\mathrm{F}$ - distance from the superior limit of IAW to the upper limbus of $\mathrm{FO} / \mathrm{fo}$. All measurements were conducted by the use of the electronic calliper (Fig. 2). Gathered results were subjected to statistical analysis with the help of MatLab computer software. In order to determine whether a given set of results is characterised by normal distribution, we used the Shapiro-Wilk test as well as the Kolmogorov-Smirnov test. In case the tested parameter did not meet the criteria of normal distribution, the Kruskal-Wallis test was used. We assumed the statistical significance of $p<0.05$.

\section{RESULTS}

Average values of the individual fragments of the IAW (according to the schematic diagram in Fig. 1D) are presented in Table 1 . The posterior part of the interatrial wall (dimension $A$ ) was 2 times smaller in the foetus group than in infants. The same correlation was observed in anterior part of the IAW (dimension C). The inferior part of the IAW (dimension D) in the foetus group was about 2.5 times smaller than in infants. In case of the superior part of the IAW (dimension F) we observed the difference about 2.2 times.

The interatrial wall size in the studied development period was greater in the horizontal than in the vertical dimension. From foetal period to infancy, the IAW size increased twice, respectively: horizontally from $10 \mathrm{~mm}$ to $18 \mathrm{~mm}$ and vertically from $7 \mathrm{~mm}$ to $14 \mathrm{~mm}$. Analysis of gathered atrial measurements revealed, that the main part of the atrial septum - foramen ovale, and after birth — fossa ovalis, is slightly larger 

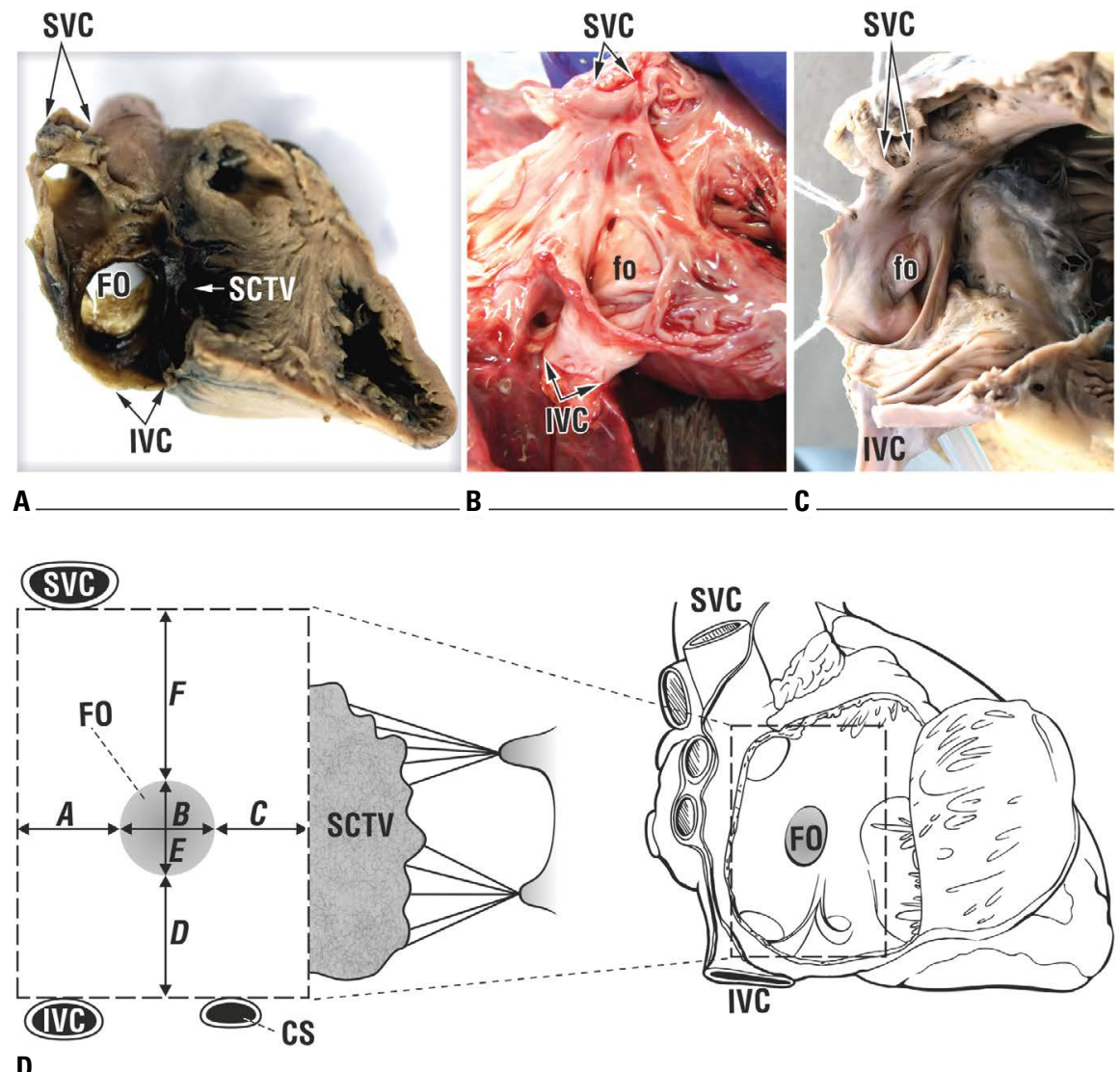

Figure 1. View from the right side of the interatrial wall; A. Human heart, $22 \mathrm{Hbd}$, male; B. Human heart, 3 weeks old, male; C. Human heart 1 year old, female; $\mathbf{D}$. The diagram of the interatrial wall. Horizontal dimensions $(A, B, C)$, vertical dimensions $(D, E, F)$; SVC - superior vena cava; IVC — inferior vena cava; FO — foramen ovale; fo — fossa ovalis; SCTV — septal cusp of the tricuspid valve; CS — coronary sinus; IAW - interatrial wall; $A$ - dimension between posterior limit of the IAW and posterior limbus of $\mathrm{FO} / \mathrm{fo}_{0} ; B$ - distance between the anterior and posterior limbus of $\mathrm{FO} / \mathrm{fo} ; C$ - distance from the anterior limbus of $\mathrm{FO} / \mathrm{fo}$ to the anterior limit of the IAW; $D$ - distance from the inferior limit of the IAW to the inferior limbus of $\mathrm{FO} / \mathrm{fo}_{0} ; E$ - distance between upper and bottom limbus of $\mathrm{FO} / \mathrm{fo}_{0} ; F$ - distance from the superior limit of IAW to the upper limbus of FO/fo.

when looking horizontally in comparison with the vertical perspective (dimensions B and E). In the group of infants the average size of the fossa ovalis from the horizontal perspective was almost $7 \mathrm{~mm}$, while in foetuses and neonates, this value was comparable and it measured about $5 \mathrm{~mm}$. Horizontal dimension of the FO/fo in all three age groups taken into account, was increasing gradually and reached approximately $4 \mathrm{~mm}, 5 \mathrm{~mm}$ and almost $7 \mathrm{~mm}$, respectively.

The percentage share of all fragments included in the total horizontal $(A+B+C)$ and vertical $(D+E+F)$ axis of the IAW are presented in Table 2. Posterior part of the IAW, back to the FO/fo (dimension A), in all age groups constituted about $25 \%$ of the horizontal IAW dimension. Anterior part of the IAW, in front of the $\mathrm{FO} / \mathrm{fo}$ (dimension C) constituted less than $30 \%$ in the group of foetuses and neonates, while in infant group its percentage increased significantly reaching
$35 \%$. In the same age group the inferior portion of the IAW (dimension D) also increased significantly (from $20 \%$ to $26 \%$ ), while the superior fragment of the IAW (dimension F) remained relatively unchanged during the individual development and accounted for approximately $26 \%$. Our data clearly demonstrated that percentage share of the superior and posterior fragments in the IAW construction, does not increase during the individual development, while its anterior and inferior parts increased their shares considerably by $8 \%$ and $6 \%$, respectively. In case of the $\mathrm{FO} / \mathrm{fo}$, percentage participation in the IAW construction (horizontal dimension) constituted $50 \%$ of its size in the foetal period and decreased reaching $39 \%$ in infants. In vertical dimension its size in foetuses and infants constituted $55 \%$ and $47 \%$, respectively.

Data describing the centre position of FO in relation to the interatrial wall are shown in Table 3 . In the 


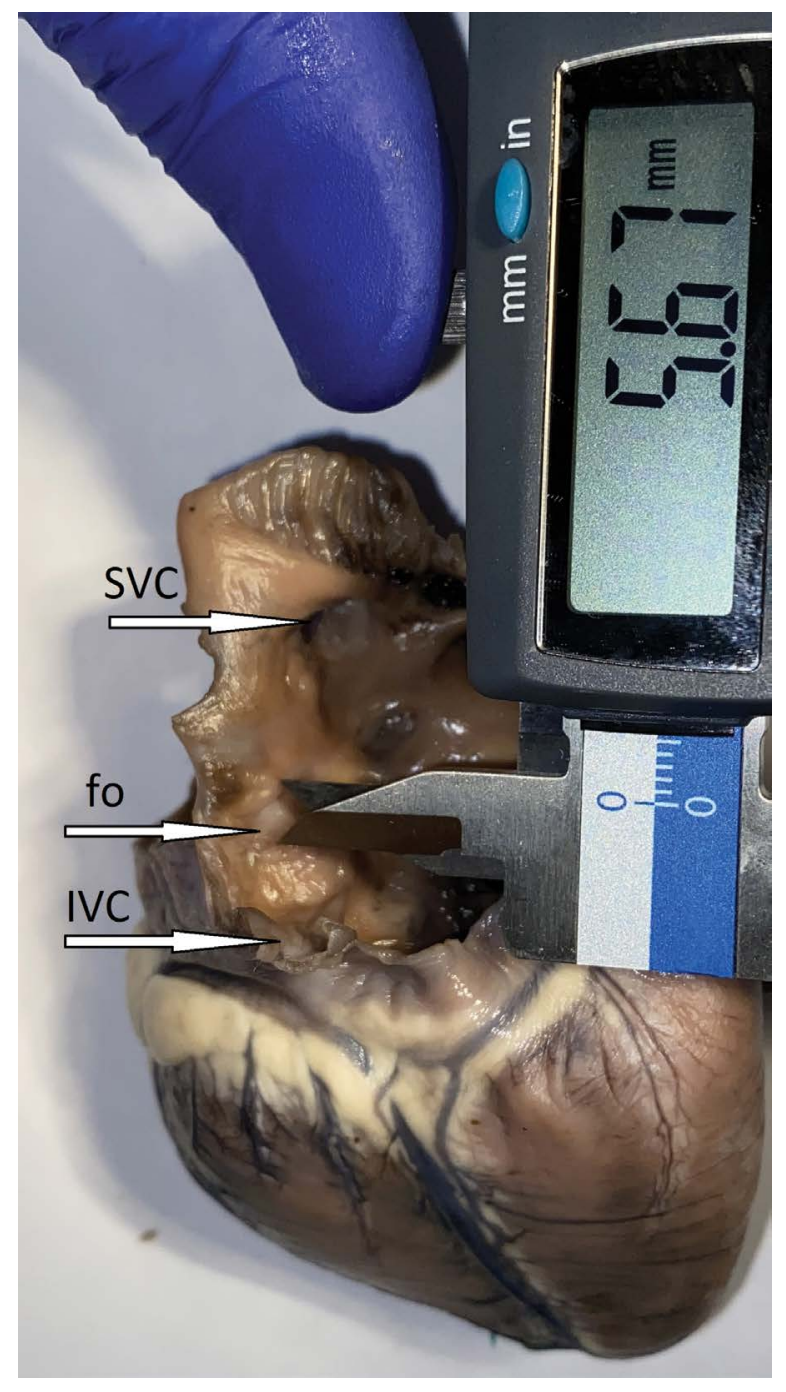

Figure 2. Method of taking measurements with electronic calliper; SVC - superior vena cava; IVC — inferior vena cava; fo — fossa ovalis.

group of foetuses as well as neonates and infants, the centre of the FO did not coincide with the centre of the IAW. These results suggest that the FO/fo centre is positioned in the infero-posterior quadrant of the IAW in foetuses group (closer to IVC) and changes toward the supero-posterior quadrant in infants (toward the SVC); however, in this case no significant statistical difference was found.

\section{DISCUSSION}

For many years the atrial septum of the heart has been the object of anatomical as well as cardiological research. The development of the interatrial wall as well as the true atrial septum during embryogenesis is quite complicated $[1,6,8,12]$. The available literature provides many interesting reports about the construction of the interatrial wall, however, almost all data comes from research carried out on the hearts of adults. With the development of new diagnostic and treatment techniques, the number of interventions performed on the heart, even during intrauterine life or shortly after childbirth, steadily increases $[3,5]$. Intracardiac procedures performed during foetal life should be reinforced not only by clinical practice, but also by the knowledge about the development and anatomical structure of the heart. Our study demonstrated that during ontogenesis, from the foetal period to infancy, the fragments of the IAW increases their dimensions individually and unevenly. For example, the part of IAW, posterior to the FO/fo, increased its absolute dimensions almost twice but its percentage share in the horizontal dimension of the IAW did not increased - remained constant. In case of the anterior part of IAW (in front of the FO/fo) we observed that its absolute dimensions also became twice as large as before but, in contrast to the posterior part of the wall, its percentage share in the total horizontal dimension of the IAW increased by about $8 \%$. Similar dependencies were observed in the vertical dimension of the septal wall, where both, its superior and inferior parts, increased its dimensions by two-fold. During this ontogenesis, the share of the superior part of the septal wall within the total vertical dimension of the IAW remained constant, while the proportion of the inferior part of the septal wall increased by about $6 \%$. Therefore, we can argue that in the early stages of life, from the foetal period to the infancy, the largest increase in the IAW construction was observed in its anterior and inferior parts. Vigneswaran et al. [13] performed the atrial septum and foramen ovale measurements on 40 foetuses in the third trimester of foetal life by performing prenatal echocardiography. They showed that FO size in the vertical dimension was on average $8.8 \mathrm{~mm}$ and its ratio in relation to the entire length of the IAW accounted for $54 \%$. In our morphological study, the absolute size of $\mathrm{FO}$ in the vertical dimension was smaller, in foetuses amounted to mean $4.5 \mathrm{~mm}$, while the ratio of the vertical dimension size of FO to the total IAW size was comparable and constituted $53.7 \%$. The observed difference in the absolute size of FO was most likely due to the fact that the material we examined was fixed in formalin, which caused a minor shrinkage of the cardiac tissue. However, similar percentual participation of $\mathrm{FO}$ in relation to the vertical dimension of the IAW described in both studies, suggests that the above differences may be indeed 
Table 1. The mean dimensions of the interatrial wall in particular age groups (1 - foetuses, 2 - neonates, 3 - infants)

\begin{tabular}{|c|c|c|c|c|c|c|}
\hline Interatrial wall dimensions [mm] & $\begin{array}{l}\text { 1. Foetuses } \\
(\mathrm{n}=41)\end{array}$ & $\begin{array}{l}\text { 2. Neonates } \\
(\mathrm{n}=30)\end{array}$ & $\begin{array}{l}\text { 3. Infants } \\
(\mathrm{n}=21)\end{array}$ & P: Group 1-2 & P: Group 1-3 & P: Group 2-3 \\
\hline A & 2.46 & 2.73 & 4.67 & $>0.05$ & $<0.05$ & $<0.05$ \\
\hline B & 5.17 & 5.20 & 6.95 & $>0.05$ & $<0.05$ & $<0.05$ \\
\hline C & 2.78 & 3.20 & 6.05 & $>0.05$ & $<0.05$ & $<0.05$ \\
\hline Horizontal dimension $(A+B+C)$ & 10.41 & 11.13 & 17.67 & & & \\
\hline D & 1.49 & 1.93 & 3.71 & $>0.05$ & $<0.05$ & $<0.05$ \\
\hline $\mathrm{E}$ & 4.07 & 4.97 & 6.71 & $<0.05$ & $<0.05$ & $<0.05$ \\
\hline $\mathrm{F}$ & 1.78 & 2.60 & 3.81 & $<0.05$ & $<0.05$ & $<0.05$ \\
\hline Vertical dimension $(\mathrm{D}+\mathrm{E}+\mathrm{F})$ & 7.34 & 9.5 & 14.23 & & & \\
\hline$E / B(\%)$ & 78.72 & 95.57 & 96.55 & & & \\
\hline Vertical/Horizontal dimensions (\%) & 70.51 & 85.35 & 80.53 & & & \\
\hline
\end{tabular}

IAW - interatrial wall; FO — foramen ovale; fo — fossa ovalis; A — dimension between posterior limit of the IAW and posterior limbus of FO/fo; B - distance between the anterior and posterior limbus of $\mathrm{FO} / \mathrm{fo} ; \mathrm{C}$ - distance from the anterior limbus of $\mathrm{FO} / \mathrm{fo}$ to the anterior limit of the IAW; D - distance from the inferior limit of the IAW to the inferior limbus of $\mathrm{FO} / \mathrm{fo}$; $\mathrm{E}$ - distance between upper and bottom limbus of FO/fo; F - distance from the superior limit of IAW to the upper limbus of FO/fo

Table 2. Percentage share of individual parts included in the total size of the horizontal $(A+B+C)$ and vertical dimension $(D+E+F)$ of the interatrial wall in individual age groups: (1 - foetuses, 2 - neonates, 3 - infants)

\begin{tabular}{lcccccc}
\hline Percentage share of individual parts of IAW & $\begin{array}{c}\text { Group 1 } \\
\text { (n= 41) }\end{array}$ & $\begin{array}{c}\text { Group 2 } \\
(\mathbf{n = 3 0 )}\end{array}$ & $\begin{array}{c}\text { Group 3 } \\
(\mathbf{n = 2 1 )}\end{array}$ & P: Group 1-2 & P: Group 1-3 & P: Group 2-3 \\
\hline A/horizontal dimension of IAW & $24 \%$ & $24 \%$ & $26 \%$ & $>0.05$ & $>0.05$ & $>0.05$ \\
B/horizontal dimension of IAW & $50 \%$ & $47 \%$ & $39 \%$ & $>0.05$ & $<\mathbf{0 . 0 5}$ & $<\mathbf{0 . 0 5}$ \\
C/horizontal dimension of IAW & $26 \%$ & $29 \%$ & $34 \%$ & $>0.05$ & $<\mathbf{0 . 0 5}$ & $>0.05$ \\
D/vertical dimension of IAW & $20 \%$ & $21 \%$ & $26 \%$ & $>0.05$ & $<\mathbf{0 . 0 5}$ & $<\mathbf{0 . 0 5}$ \\
E/vertical dimension of IAW & $55 \%$ & $53 \%$ & $47 \%$ & $>0.05$ & $<\mathbf{0 . 0 5}$ & $<\mathbf{0 . 0 5}$ \\
F/vertical dimension of IAW & $25 \%$ & $26 \%$ & $27 \%$ & $>0.05$ & $>0.05$ & $>0.05$ \\
\hline
\end{tabular}

IAW - interatrial wall; FO — foramen ovale; fo — fossa ovalis, A — dimension between posterior limit of the IAW and posterior limbus of FO/fo; B - distance between the anterior and posterior limbus of $\mathrm{FO} / \mathrm{fo}_{0} ; \mathrm{C}$ - distance from the anterior limbus of FO/fo to the anterior limit of the IAW; D - distance from the inferior limit of the IAW to the inferior limbus of $\mathrm{FO} / \mathrm{fo}_{0}$; $\mathrm{E}$ - distance between upper and bottom limbus of FO/fo; F - distance from the superior limit of IAW to the upper limbus of FO/fo

Table 3. The position of the centre of the foramen ovale and fossa ovalis within the interatrial wall in the vertical and horizontal dimension in particular age groups

\begin{tabular}{|c|c|c|c|}
\hline & $\begin{array}{c}\text { Foetuses } \\
(n=41)\end{array}$ & $\begin{array}{c}\text { Neonates } \\
(n=30)\end{array}$ & $\begin{array}{l}\text { Infants } \\
(n=21)\end{array}$ \\
\hline Horizontal: $(C+0.5 B) /(A+B+C)$ & 0.51 & 0.52 & 0.54 \\
\hline Vertical: $(D+0.5 E) /(A+B+C)$ & 0.48 & 0.47 & 0.50 \\
\hline \multicolumn{4}{|c|}{$\begin{array}{l}\text { IAW - interatrial wall; } F 0 \text { - foramen ovale; fo - fossa ovalis; } A-\text { dimension be- } \\
\text { tween posterior limit of the IAW and posterior limbus of } \mathrm{FO} / \mathrm{fo} ; \mathrm{B}-\text { distance between } \\
\text { the anterior and posterior limbus of } \mathrm{FO} / \mathrm{fo} ; \mathrm{C}-\text { distance from the anterior limbus of } \mathrm{FO} / \mathrm{fo} \\
\text { to the anterior limit of the IAW; } \mathrm{D}-\text { distance from the inferior limit of the IAW to the } \\
\text { inferior limbus of } \mathrm{FO} / \mathrm{fo} ; \mathrm{E}-\text { distance between upper and bottom limbus of } \mathrm{FO} / \mathrm{fo} \text {; } \\
\mathrm{F} \text { - distance from the superior limit of IAW to the upper limbus of FO/fo }\end{array}$} \\
\hline
\end{tabular}

attributable to the properties of tissue-preserving fluid, acting on the structure of the tested material.

Analysis of our results let us conclude that during the individual development from the foetal period to the infancy, the FO/fo is modestly larger horizontally than in its vertical perspective. In the foetus group, the average FO size in the horizontal dimension was approximately $5 \mathrm{~mm}$, while in infants, fo was $2 \mathrm{~mm}$ larger. Vertical dimension of $\mathrm{FO} / \mathrm{fo}$ in turn, increased from about 4 to less than $7 \mathrm{~mm}$ within this timescale. In the study of Kucybała et al. [4] we can find the similar tendency, that horizontal dimension of the fo is slightly bigger than vertical one. In their work the mean antero-posterior and cranio-caudal diameters of the fo were: $14.1 \mathrm{~mm}$ and $12.1 \mathrm{~mm}$, respectively. Interestingly, the study by Joshi et al. [2], where 50 adult hearts were taken into account, demonstrated the same relation in fo dimensions: the horizontal dimension was shown to be greater than vertical: $14.5 \mathrm{~mm}$ and $12.6 \mathrm{~mm}$, respectively. In addition, Joshi et al. [2] stated that in as many as $82 \%$ of the examined hearts, fo was of oval shape and the remaining ones were round. Similar results regarding the shape 
of the fossa ovalis were obtained by Reig et al. [11] who analysed the atrial septum in 100 human hearts. Their data show that the dominant, oval shape of fossa ovalis was found in $78 \%$ of examined hearts while in other cases the one was round. Somehow relating to the above we demonstrated that in both, foetal and infant developmental periods, more than $70 \%$ of hearts were characterised by the presence of oval-shaped FO and fo, respectively. As for the FO and fo changing with time, our analysis of development of the ones indicated that during foetal life it constituted the largest part of the septum while after birth, its share in the interatrial wall construction gradually decreased. While in foetuses it made up for more than $50 \%$ of the IAW size in both, vertical and horizontal dimension, in the subsequent months of ontogenesis, its share was significantly decreasing. In infants its size constituted about $39 \%$ of the horizontal dimension and $47 \%$ of the vertical dimension. Decrease in the share of FO and fo in the IAW construction resulted from increased as well as uneven growth of individual IAW parts and this process was particularly noticeable in the inferior and anterior part of the atrial septal wall. We can therefore conclude that in all three groups, foetuses as well as neonates and infants, the centre of the FO and fo, respectively does not coincide with the centre of IAW. On the basis of our data we can assume that the FO centre tends to be found in the infero-posterior quadrant of the IAW (closer to IVC) but fo centre is mostly in the supero-posterior quadrant, however, this tendency is not statistically significant.

\section{CONCLUSIONS}

Our study demonstrated that during ontogenesis, from the foetal period to infancy, the parts of the IAW increase their dimensions unevenly. The FO growth is smaller, compared to the rest of the IAW development. Our analysis of FO development indicated that during foetal life it constituted the largest part of the septum, while after birth, the fossa ovalis share in the IAW construction gradually decreased. Moreover, we can assume that the FO centre tends to be found in the postero-inferior quadrant of the IAW but fossa ovalis in postero-superior quadrant of the IAW. It seems that understanding the processes taking place in particular parts of IAW at various stages of individual development, potentially earliest possible, may facilitate making key decisions as to the appropriate timing to introduce given medical intervention and the manner of its implementation.

\section{REFERENCES}

1. Jensen B, Spicer DE, Sheppard MN, et al. Development of the atrial septum in relation to postnatal anatomy and interatrial communications. Heart. 2017; 103(6): 456-462, doi: 10.1136/heartjnl-2016-310660, indexed in Pubmed: 28003417.

2. Joshi SD, Chawre HK, Joshi SS. Morphological study of fossa ovalis and its clinical relevance. Indian Heart J. 2016; 68(2): 147-152, doi: 10.1016/j.ihj.2015.08.001, indexed in Pubmed: 27133322.

3. Kalish BT, Tworetzky W, Benson CB, et al. Technical challenges of atrial septal stent placement in fetuses with hypoplastic left heart syndrome and intact atrial septum. Catheter Cardiovasc Interv. 2014; 84(1): 77-85, doi: 10.1002/ccd.25098, indexed in Pubmed: 23804575.

4. Kucybała I, Ciuk K, Klimek-Piotrowska W. Clinical anatomy of human heart atria and interatrial septum - anatomical basis for interventional cardiologists and electrocardiologists. Part 1: right atrium and interatrial septum. Kardiol Pol. 2018; 76(3): 499-509, doi: 10.5603/KP.a2017.0248, indexed in Pubmed: 29350387.

5. Mackesy MM, Kalish BT, Tworetzky W, et al. Sonographic pulmonary abnormalities in fetuses with hypoplastic left heart syndrome and intact atrial septum undergoing attempted atrial septostomy in utero. Ultrasound Q. 2017; 33(1): 82-85, doi: 10.1097/RUQ.0000000000000247, indexed in Pubmed: 27575842.

6. Moore KL, Persaud TV. The cardiovascular system. In: The Developing Human, Clinically Oriented Embryology. 7th ed. Saunders, Elsevier, Philadelphia, Pennsylvania 2003: 340-345.

7. Padilla T, Zapata M, Díaz LH, et al. Results of balloon atrial septostomy as preparation for surgical correction in transposition of great arteries. World J Pediatr Congenit Heart Surg. 2011; 2(2): 249-252, doi: 10.1177/2150135110395409, indexed in Pubmed: 23804980.

8. Paulsen F, Waschke J. Thorax. In: Sobotta, Atlas der Anatomie des Menschen. Innere Organe. 23th ed. Urban \&Fischer 2010: 6-7.

9. Rashkind W. Creation of an atrial septal defect without thoracotomy. A palliative approach to complete transposition of great arteries. JAMA. 1966; 196(11): 991-992, doi: 10.1001/jama.1966.03100240125026.

10. Rashkind WJ. Transcatheter treatment of congenital heart disease. Circulation. 1983; 67(4): 711-716, doi: 10.1161/01.cir.67.4.711, indexed in Pubmed: 6825227.

11. Reig J, Mirapeix R, Jornet A, et al. Morphologic characteristics of the fossa ovalis as an anatomic basis for transseptal catheterization. Surg Radiol Anat. 1998; 19(5): 279-282, doi: 10.1007/s00276-997-0279-0.

12. Sadler TW. Cardiovascular system. In: Langman's Medical Embryology. 12th ed. Lippincott Williams \& Wilkins, Philadelphia 2013: 171-177.

13. Vigneswaran TV, Zidere V, Miller OI, et al. Usefulness of the prenatal echocardiogram in fetuses with isolated transposition of the great arteries to predict the need for balloon atrial septostomy. Am J Cardiol. 2017; 119(9): 1463-1467, doi: 10.1016/j.amjcard.2017.01.017, indexed in Pubmed: 28283176. 